\title{
Infectious cancers
}

\author{
Robert M. Hurwitz ${ }^{1}$
}

1 Dermatopathology, Indianapolis, Indiana, USA

Key words: cancer, infectious, cancer, myth, melanoma

Citation: Hurwitz RM. Infectious cancers. Dermatol Pract Concept 2015;5(3):13. doi: 10.5826/dpc.0503a13

Copyright: $\odot 2015$ Hurwitz. This is an open-access article distributed under the terms of the Creative Commons Attribution License, which permits unrestricted use, distribution, and reproduction in any medium, provided the original author and source are credited.

Acknowledgement: Thanks to Steven A. Hurwitz, MS, MBA, for his active support and assistance.

Corresponding author: Robert M. Hurwitz, MD, 1829 Box Elder Court, Indianapolis, IN, 46260 USA. Email: bobbybur@aol.com

\section{Patient's question}

"Doctor, please wait before rushing off. I have a question for you. Do I have cancer? Please be direct and honest with me, and explain to me the truth about what you know about cancer in a manner that I, as a layman, will be able to understand what you tell me. Most importantly, what is cancer, and what causes cancer? If I have cancer, how long will I live, and will I have just a little or significant pain? And lastly, is there any chance of a cure"?

\section{Doctor's answer}

"I don't know! No one knows the answers to your questions with absolute certainty. You must now excuse me I have other patients to see."

\section{Discussion}

Cancer is a general term frequently used to describe a variety of malignant altercations, most of which manifest invasiveness and metastasize to multiple sites, resulting in progressive illness and death [1]. For centuries, the causes of a variety of malignant conditions and cancer were unknown; some cases even were considered mythical, such as "the black plague," which was attributed at one time to an ethnic group, i.e., the Jews. This mythical concept, for example, resulted in the extermination of an enormous number of Jews. Only decades later did this mythical misconception prove false, and not sur- prisingly, found to be caused by infectious gram negative bacteria, Yersinia pestis, by Selman Waksman [2] and his protégé Albert Schatz, in the 1940s and early 1950s. The black plague was easily and quickly controlled and minimized by the use of streptomycin, a gray secreted substance from an actinomyces, Actinomyces griseus (gray). Interestingly, my first and only experience with the black plague was at the young age of 27 in March 1969 while in the United States Navy, 3rd Medical Battalion of the $3^{\text {rd }}$ Marine Division, where I treated an epidemic of plague in the village of Pho Hoi, Viet Nam. I documented 38 men, women and children with classic signs and symptoms of plague including prominent buboes, which were treated and resolved with a combination of streptomycin and tetracycline. It was hard for me to believe that the life-saving drug streptomycin had been available for only 25 years. Complete clearing of the plague was ensured in days after treatment was instituted. Not surprisingly, the only ones that were exterminated then were the carrier rodents.

Although melanoma is considered an unquestionable malignant growth, i.e., a cancer, its etiology to date remains undefined or uncertain, although sun exposure is considered strongly [3]. To support UVR exposure to some melanomas, there are a variety of unpredictable chemotherapeutic and immunotherapeutic treatment regimens such a dacarbazine (DTIC) and interleukin-2 (HDIL-2 of interferon). "Novel and Emerging Therapies for Melanoma" [4] acknowledges variable responses and unfortunately significant toxicity. Other therapies in use and under investigation include targeted immunotherapy and or immunotherapy, vemurafenib and ipilimumab. 
To date, none of these modalities appear to be directly related to an infectious organism or directly eliminate or cure a melanoma. Interestingly, today many believe emphatically that the sun with its ultraviolet rays is the offending agent; even though melanoma is not uncommonly present in non-sun-exposed areas of the body, including the genitalia, oral/nasal mucosa, perianal, palm/soles, and regions subungual. In addition, even the renowned ABCD's (Asymmetry, Border, Color and Diameter), once considered diagnostically infallible for a diagnosis of melanoma clinically are now breaking down among those arched backers, who now recognize numerous benign and malignant melanocytic proliferations, as well as pigmented and non pigmented and non melanocytic proliferations that simulate the ABCD's. Frequent strong and obnoxious comments have been expressed commonly by colleagues towards Dr. A. Bernard Ackerman's views, especially on the subject of melanoma. As quoted in The Sun and the "Epidemic" of Melanoma: Myth on Myth! Ackerman [3] responded straightforwardly, honestly, unswervingly, directly and poignantly from the film Breaker Morant, "Scientists are human; they do not wait for proof; many devote their professional lives to seeking evidence for hypotheses (especially well-funded hypotheses) they've chosen to believe." [5]

An extraordinary, informative and exceptionally brilliant must read book is The Emperor of All Maladies: A Biography of Cancer, by Siddhartha Mukherjee [6]. In his work, Mukherjee attacks cancer and outlines the morbid history in detail and the unbelievable events that physicians, investigators and researchers had to deal with in their study of cancer.

For a decade, from 2003-2013, Clifton Leaf conducted an extensive investigation and introspection on the war on cancer, which is published in his book The Truth in Small Doses: Why We're Losing the War on Cancer-and How to Win It [7]. He understood that science determines the limits of the possible and that engineering allows us to reach them. He was well aware of the difficulty to secure funding in an atmosphere of competition overriding collaboration. His investigations extended to include, among others, the tribulations of Denis Burkitt, the Epstein-Barr virus, and the complexities of oncogenes.

Recently, in Claudia Cornwall's [8], Catching Cancer: The Quest for Its Viral and Bacterial Causes, there were astonishing, hard to believe, incredible accounts of the individual trials and tribulations that researchers were forced to go through to prove their theories that an infectious agent was the cause of the cancer at hand. Funding was nearly out of the question to obtain. One such group (Marshall and Warren) drank a bacterial impregnated liquid to prove their point that $H$. pylori bacteria resulted in gastric ulcers (and later gastric carcinoma). There were successful investigators who associated an infectious agent with cancer, who included the likes of:

- Denis P. Burkitt (Burkitt's lymphoma)
- R. Palmer Beasley (hepatitis B and liver cancer)

- Harald zur Hausen (human papillomavirus HPV 16 and cervical squamous cell carcinoma)

- Barry Marshall and Robin Warren (H. pylori gastric ulcer and gastric cancer)

- Patrick S. Moore and Yuan Chang (Merkel cell cancer with the polyomavirus)

- Yuan Chang, Ethel Cesarman, and Melissa S. Pessin (AIDS/ Kaposi sarcoma, now considered by some to be a hyperplasia)

- Ludwik Gross, Paul Ewald, Cynthia L. Sears, Robert Holt (stealth and colonic infection and cancer; "fusobacterium bacterimia nucleatium")

An interesting twist to the infectious etiology of cancers concerns the fact that the same infectious agent may be implicated and proliferates in a variety of clinical expressions. Some presentations included those simulating an inflammatory reaction. For example, lymphomatoid papulosis is an inflammatory variation of a CD 30 Lymphoma, other infectious agents often prove to be viral or bacterial organisms such as the human papillomavirus (HPV), Epstein-Barr virus (herpes virus (infectious mononucleosis, and lymphoma), and HIV and lymphoma, hepatitis B virus and liver cancer, herpes virus, Bartonella henselae, and Borrelia (spirochete). Similarly, inflammatory HIV, viral hepatitis, vaccines, and drugs such as TNF- $\alpha$ inhibition, gold and/or interferon injection may result in clinical inflammatory reactions such as granuloma annulare as well as lymphoproliferative/hematopoietic malignancies [9].

It is common knowledge that it is usual for an infectious agent to alter its normal mechanisms of a molecular cellular proliferation, and/or its molecular pathway. On the other hand, there are significant genomic and molecular studies in progress showing alternation of chromosomes and altered genetic pathways in both benign and malignant conditions. The question becomes just what alteration an infectious organism may assume. Just because an organism may or may not be identifiable per se by culture or electron microscopy, or any other investigative mechanism, does not necessarily purport that they are present or not present, active and/or out of action. Understanding that some cancers have been found to be associated with an infectious agent is in reality a fact, and not only for the classification of cancers.

In brief summary, there are numerous cancers such as ovarian, breast, brain, pancreatic and melanoma with unknown etiologies, and yet today there are many progressive inflammatory and neoplastic diseases of known infectious etiology. As a consequence, an infectious etiology must continue to be a main concern. Some conditions on the other hand, might well be expected to be infectious, such as amyotrophic lateral sclerosis (Lou Gehrig's disease) and Alzheimer's disease, which as yet are undetermined. 
During the past decade there has been a surge in the literature strongly associating infectious agents with cancers of various types, as previously described. Some therapeutic modalities have been found to be effective in eliminating some cancers completely, or at least minimizing the consequences from the cancer. New and sophisticated therapeutic modalities, specifically attacking the infectious agent or their consequences have been and are being discovered and used to affect the infectious agent directly and to minimize their secondary effects: "cancer-control measures . . . avoid attempting to completely eradicate tumor cells, by aiming to (i) limit essential resources to cancer cells, (ii) disrupt cooperation among cancer cells; and (iii) prevent host damage." [10] In the meantime, study of neoplastic infections agents will continue to illuminate molecular oncogenic processes. Both known and unidentified infectious agents have yet to be implicated in human cancer. When all is said and done and all things considered, the future looks bright, but infectious etiology must not be ignored or overlooked. We can and should expect a proliferation of new therapeutic agents, e.g., vaccines, [11] in association with one cancer or another [12].

\section{References}

1. Stedman's Medical Dictionary. Baltimore, MD: The Williams \& Wilkins Company, 1961.

2. Heynick F. Jews and Medicine: An Epic Saga. Hoboken, NJ: Ktav Publishing House, Inc., 2002.
3. Ackerman AB. The Sun and the "Epidemic" of Melanoma: Myth on Myth! Contrary View on Behalf of Patients, $2^{\text {nd }}$ ed. New York, NY: Ardor Scribendi, Ltd., 2008.

4. Novel and Emerging Therapies for Melanoma. TargetedOnc. com. March 5, 2014. Intellisphere LLC. Accessed March 11, 2015. http://www.targetedonc.com/articles/novel-and-emergingtherapies-for-melanoma/1

5. Jenkins HW Jr. "The science of Gore's Nobel”. The Wall Street Journal, December 5, 2007.

6. Mukherjee S. The Emperor of All Maladies. A Biography of Cancer. New York, NY: Simon \& Schuster, 2011.

7. Leaf C. The Truth in Small Doses: Why We're Losing the War on Cancer-and How to Win It. New York, NY: Simon \& Schuster, 2013.

8. Cornwall C. Catching Cancer: The Quest for Its Viral and Bacterial Causes. Lanham, MD: Rowman \& Littlefield Publishers, 2013

9. Shinkai K, Rosenbach M, Wintroub B, Berger G. Therapeutic Strategies in Dermatology: Granuloma annulare. Derm101.com. Accessed March 11, 2015.http://www.derm101.com/therapeuticl granuloma-annulare-2/key-points/

10. Jansen G, Gatenby R, Aktipis CA. Opinion: Control vs. eradication: Applying infectious disease treatment strategies to cancer. PNAS 2015;112:937-8. doi: 10.1073/pnas1420297111.

11. Srivastava A, Shirwan H. Armed with the right adjuvant system, vaccines are poised to tackle on of the world's most intractable diseases. The-Scientist.com. March 31, 2015. LabX Media Group. http://www.the-scientist.com/?articles.view/articleNo/42563/title/ Opinion-Making-Cancer-Vaccines-Work/

12. Pagano HS, Blasner M, Buedia MA, et al. Infectious agents and cancer: criteria for a causal relation. Semin Cancer Biol 2004;14(6):453-71. 